\title{
VASCULAR SURGERY
}

\section{Day of surgery: the impact on patient length of stay in a tertiary vascular unit}

\author{
NC Holford, C Ní Ghuidhir, L Hands
}

Vascular Department, Oxford University Hospital, Oxford, UK

\section{ABSTRACT}

BACKGROUND Our hypothesis was that patients undergoing surgery earlier in the week would have better access to physiotherapy and other discharge services after surgery and, as a result, would have a shorter length of hospital stay compared with patients undergoing surgery later in the week. This study aimed to assess whether there is a significant difference in postoperative length of hospital stay between the groups with secondary assessment by operation subtype.

METHODS We identified all patients admitted for vascular surgery in 2015 from a prospectively collected database and divided the week into Monday to Wednesday and Thursday to Friday. Endovascular cases were included but day cases were excluded. Further analysis was performed with a breakdown in both groups by operation type. Statistical analysis was performed using SPSS version 16.0.

RESULTS We identified 652 patients who met our criteria. Within the elective patient group, there was a significantly longer length of stay of three days for the late-week group compared with two days for the early-week group $(P=0.016)$. Femoral artery procedures had a median length of stay of two days for those operated on early in the week compared with four days later in the week $(P<0.005)$. Open abdominal aortic aneurysm repair showed a trend to longer length of stay in the late-week group $(P=0.06)$.

CONCLUSION Day of surgery appears to impact on patients' length of stay following vascular procedures, with the greatest impact on medium-sized procedures. This difference could be explained by the difference in weekend support services, but further evaluation is required following introduction of weekend support services to assess this.

\section{KEYWORDS}

Hospital stay - Time management - Vascular surgical procedure

Accepted 21 April 2019

\section{CORRESPONDENCE TO}

NC Holford, E: Nicholas.holford@doctors.org.uk

\section{Introduction}

Our experience suggested that patients operated on later in the week might have a longer length of hospital stay than those who underwent surgery earlier in the week. We decided to investigate whether this was correct as a step towards reducing the length of stay, possibly by introducing seven-day working for physiotherapy and occupational therapy services. Enhanced recovery programmes have provided growing evidence that a shorter length of stay is cost saving to the hospital, ${ }^{1}$ with decreased morbidity and no clear evidence of increased readmissions across multiple specialties. ${ }^{2-4}$ Previous studies in vascular units have shown that most delayed discharges are in elderly patients with complex rehabilitation and discharge requirements and this accounted for an extra 19\% of hospital bed days over the expected length of stay. ${ }^{5}$ It has been shown that a reduced stay does not result in a higher readmission rate for patients undergoing open and endovascular procedures for peripheral arterial disease. ${ }^{6}$
In our unit at Oxford University Hospital, patients undergoing elective vascular procedures are assessed preoperatively by the anaesthesia team, optimised for surgery and admitted on the day of procedure. Patients undergoing elective open abdominal aortic aneurysm repairs are assessed with a cardiopulmonary exercise test. Open abdominal aneurysm repair patients are admitted overnight to intensive care for observation before stepping down to the ward the next day if appropriate. All other vascular procedure patients are managed postoperatively on the vascular ward unless there are individual requirements for intensive care or high dependency unit support. Patients are encouraged to ambulate following surgery and the physiotherapists are experienced with amputee rehabilitation. Our unit currently does not offer seven-day access to physiotherapy or occupational therapy services, but does have daily consultant ward rounds and support from the acute pain and diabetic teams.

Specialist surgery units have shown that patients operated later in the week have an increased length of stay 
compared with the early-week group for neurosurgical and orthopaedic patients. $^{7,8}$ The orthopaedic study used a healthcare system database of 11,965 patients undergoing primary hip or knee surgery. They found a longer length of stay for patients operated later in the week after univariate and multivariate regression, including severity of illness score and age. However, a study assessing day of surgery in patients undergoing minimally invasive lumbar fusion found no correlation in 438 patients analysed. ${ }^{9}$ This difference may reflect recovery course following different procedures in addition to the support services required before discharge. It has been demonstrated that the addition of a single extra day of rehabilitation work on a Saturday can reduce the length of hospital stay, while improving functional independence and quality of life measures in medical patients. ${ }^{10,11}$ The high rate of comorbidities common among vascular patients could make them a group that could have similar benefit from the implementation of greater weekend support. Reducing the postoperative length of stay could improve bed use, could be cost effective and could improve patient care.

\section{Methods}

A prospectively collected database was used to identify all patients admitted in 2015 under the vascular team. The database is maintained by the vascular team and operative details are recorded at time of procedure, together with dates of admission, surgery and discharge. Each operating list is allocated to a specific consultant team, with the exception of the Friday operating list, which is used by all consultant teams. We excluded daycase and non-operative patients. Patients undergoing elective and scheduled procedures were analysed together with and separately from emergency and urgent cases, as the former cases would allow for advanced planning of theatre list configuration. The patients were grouped according to the nature of their surgery. If a patient had undergone more than one procedure during their admission, then the major procedure was counted for analysis with the lesser procedures excluded. This was to stop a small procedure being unfairly skewed with a long length of stay. For example, in a patient undergoing femoral endarterectomy with iliac angioplasty, the length of stay would be largely determined by the endarterectomy rather than the angioplasty.

Patients' length of stay was compared across the week with the early week chosen as Monday to Wednesday, and Thursday and Friday counting as the late week. Analysis was performed for all patients meeting the above criteria with subgroup analysis relating to operation type. Statistical analysis was performed with SPSS 16.0, using the chisquare and Mann-Whitney U tests. Primary outcomes were patient length of hospital stay for all planned procedures and for all procedures in the early- and late-week groups. Secondary outcomes were the difference between different operation subtypes. A $P$-value less than 0.05 was used to determine significance.

In case differences in length of stay were due to differences in morbidity between patients operated in the early- or late-week groups, we compared patient comorbidities using the generated coding data for the femoral procedures from the healthcare resource group.

\section{Results}

We identified 652 patients admitted in 2015 having surgery between Monday to Friday exclusively, having excluded those who were day cases. This included 402 elective or scheduled patients and 250 emergency or urgent cases. The breakdown by operation type is shown in Figure 1 . The baseline characteristics for all patients are shown in Table 1, with a further breakdown for patient undergoing a femoral endarterectomy or bypass procedure.

From the healthcare resource group data there was a trend towards late-week patients having a higher proportion of comorbidities recorded $(0.68$ vs 0.88$)$ and this did reach significance $(P=0.02)$. The relatively low rate of comorbidities in the early-week group raised concerns, so a review was performed of all patients who underwent a femoral endarterectomy or bypass, which found similar levels of comorbidities ( $92 \%$ vs $98 \%$ ) in the two groups, with no statistical difference $(P=0.23)$. There was no clear link between the cases that were incorrectly coded and this seems to be a common occurrence. ${ }^{12}$

A further review of patient notes was performed for this group with the comorbidities at admission recorded in Table 1. The proportion of patients in the late-week group with a diagnosis of coronary artery disease was significantly higher than the early-week group. This does not seem to correlate with higher-risk patients being operated in the late-week group, as there was no difference with other comorbidities or with American Society of Anesthesiologists status $(P=0.64)$. There was a significantly higher proportion of vascular specialist anaesthesia consultants for the late-week group compared with the early-week group (0.84 vs $0.67, P=0.039)$. This may reflect the timetabling of the anaesthesia department. There was no correlation between attendance of a specialist vascular anaesthetist and a shorter length of stay. For primary operating surgeon, there was a significant correlation between longer operation duration and a consultant-led procedure $(P=0.02)$, probably reflecting the selection of appropriate cases for training. There was no difference between the proportion of consultant-led procedures between the early- or late-week groups and there was no correlation with length of stay and consultant-led procedure $(P=0.82)$. There was inconsistent recording of intraoperative blood loss, but there was no difference in recorded haemoglobin drop from laboratory measurements or need for transfusion between the early- and late-week groups.

Operation duration was used as a marker of procedure difficulty and was found to correlate strongly with length of stay $(P<0.005)$, which corresponds to the expectation that a more complex and lengthy procedure would lead to a longer recovery period. Procedures in the late-week group took significantly longer than in the early-week group (135 minutes vs 120 minutes, $P=0.012$ ). It is difficult to know 


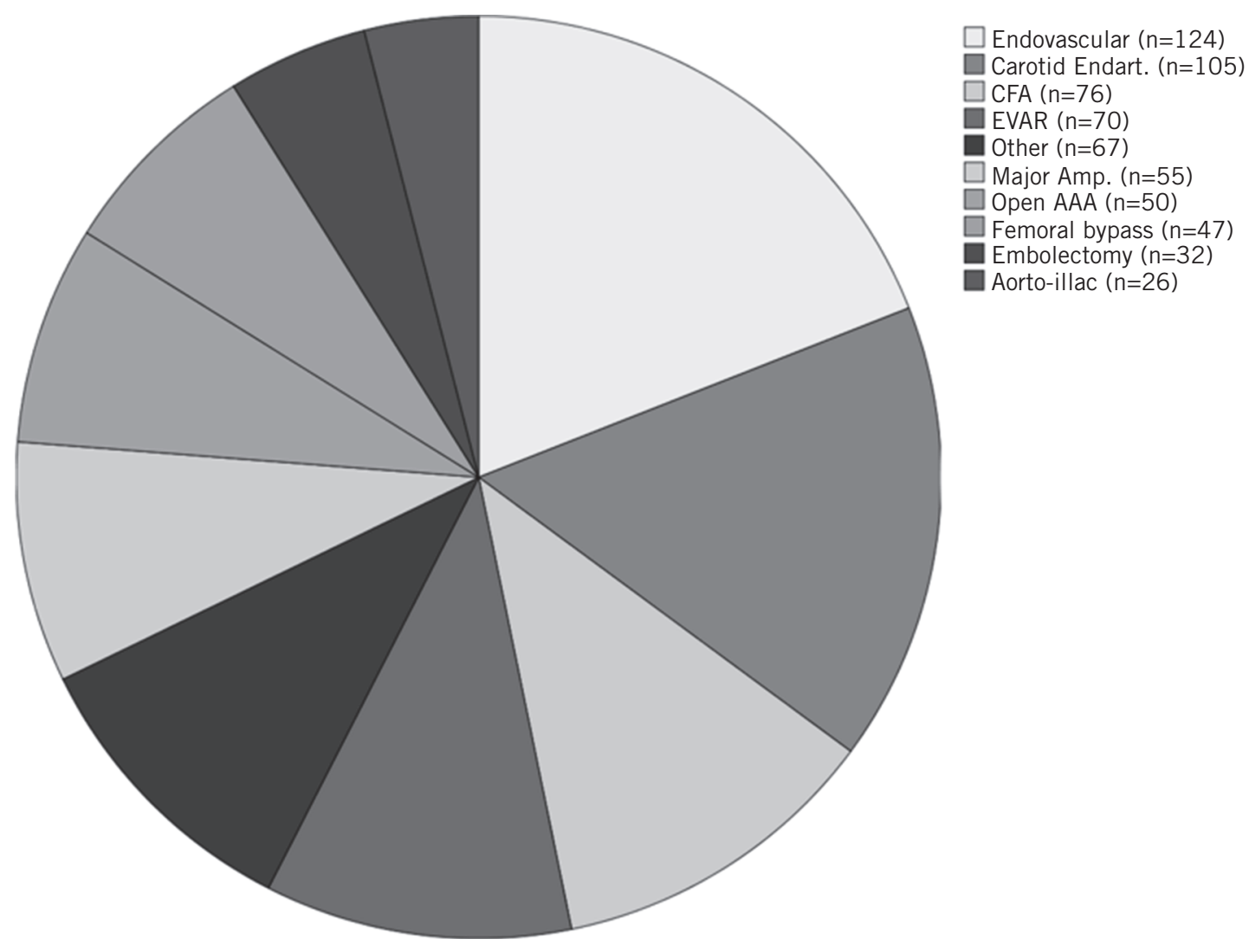

Figure 1 Proportion of patients undergoing different procedures. (endart. = endarterectomy; CFA = common femoral endarterectomy; Major Amp. = above and below knee amputations).

Table 1 Baseline characteristics of all patients with breakdown for all patients, elective patients, patients undergoing femoral procedure (including femoral endarterectomy, femoral-femoral crossover and femoral bypass procedures) and emergency patients.

\begin{tabular}{|c|c|c|c|c|}
\hline Surgery type & Characteristic & Early-week group & Late-week group & $P$-value \\
\hline \multirow[t]{2}{*}{ All patients $(n=652)(\%)$} & Percentage male & $71(n=379)$ & $68(n=273)$ & 0.34 \\
\hline & Age (years) & 72 & 73 & 0.22 \\
\hline \multirow[t]{2}{*}{ Elective and scheduled $(n=402)(\%)$} & Percentage male & $76(n=242)$ & $70(n=160)$ & 0.21 \\
\hline & Age (years) & 73 & 74 & 0.18 \\
\hline \multirow[t]{6}{*}{ Femoral procedures $(n=102)(\%)$} & Percentage male & $69(n=52)$ & $70(n=50)$ & 0.97 \\
\hline & Age (years) & 68 & 72 & 0.12 \\
\hline & Smokers & $52 \%$ & $61 \%$ & 0.32 \\
\hline & Diabetes & $38 \%$ & $35 \%$ & 0.74 \\
\hline & Coronary artery disease & $38 \%$ & $56 \%$ & 0.035 \\
\hline & Chronic kidney disease & $25 \%$ & $19 \%$ & 0.47 \\
\hline \multirow[t]{2}{*}{ Urgent and emergency $(n=250)(\%)$} & Percentage male & $63(n=137)$ & $65(n=113)$ & 0.77 \\
\hline & Age (years) & 71 & 73 & 0.50 \\
\hline
\end{tabular}


why the procedures were taking longer in the late-week group or the clinical impact of an average additional 15 minutes in the context of a two-hour operating time. When comparing patients undergoing only femoral related surgery, there was no difference in operation duration $(P=$ $0.62)$.

\section{All patients}

Across the week, there was a weak trend to longer stays for patients operated in the late-week group but it did not meet significance. The median for both groups was four days with a skew towards a longer length of stay in the late-week group compared with the early-week group.

\section{Elective and scheduled patients}

From the planned surgical work, there was a median length of stay of two days in the early-week group compared with three days for the late-week group $(P=0.016$; fig 2).

\section{Breakdown by surgery type}

A breakdown by type of surgery (Table 2) revealed a significantly increased length of stay in the late-week group compared with the early-week group for femoral procedures (Table 3). This group is significant, as they represent $25 \%$ of the planned workload and an even greater proportion of the annual patient stay. There was no significant difference in the proportion of emergency or urgent femoral cases performed between the early- and late-week groups.

Other operation types had an association but did not reach significance, though open abdominal aortic aneurysm did have a trend towards increased length of stay for the late-week group. A further breakdown is provided for endovascular cases (Table 3), and there was no significant

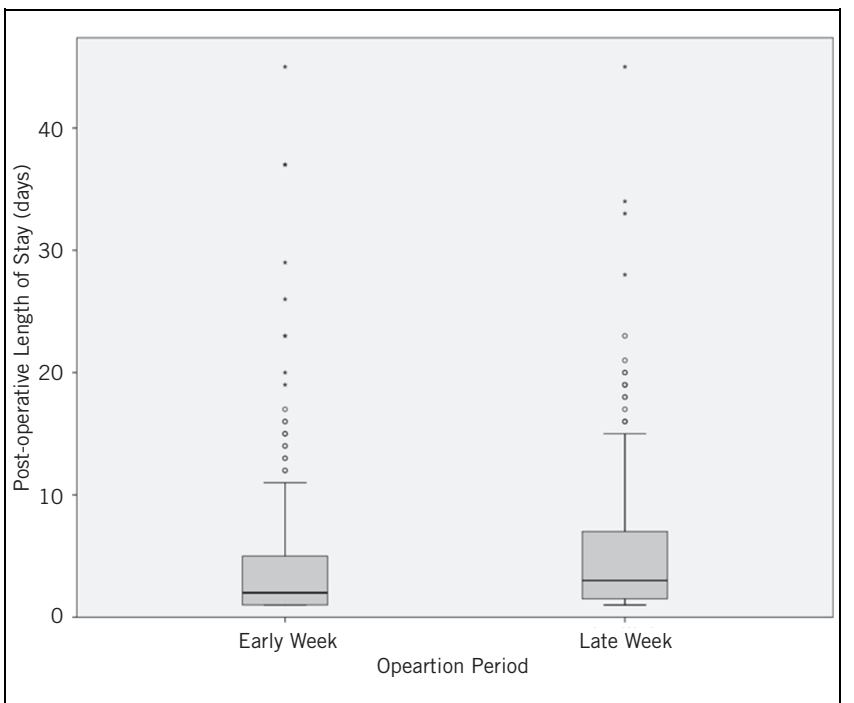

Figure 2 Length of postoperative hospital stay for patients undergoing elective or scheduled surgery, all procedures. difference between procedure times between the earlyand late-week groups $(P=0.35)$. If there was intervention at more than one level this was included as the most distal in addition to the multilevel group.

Patients undergoing femoral procedures who required a delayed major amputation $(n=8)$ had a median length of stay of 37 days compared with 3 days for patients requiring no further surgery $(P<0.005)$. Patients requiring phalanx amputations, wound debridement and fasciotomies $(n=4)$ had a median length of stay of 12 days $(P=0.05)$. There was no correlation between length of stay and patients having had prior minor or major amputations and no difference in proportion of patients needing further surgery in the early- or late-week groups. Once patients requiring further surgery were removed, the significantly increased length of stay for patients operated in the late-week group remained for both femoral endarterectomy $(n=49)$ and femoropopliteal bypass $(n=22)$ patients (Table 4; fig 3$)$.

The femoral-distal bypass early-week group had a seven-day longer length of stay compared with the lateweek group. This value is skewed by two of the four patients having the longest and third longest length of stay. There was no common factor for these two cases to explain this difference. The remaining femoral procedure numbers were too small to give meaningful results.

\section{Emergency and urgent operating}

A further breakdown was performed for urgent and emergency operating based on operating period. There was a trend towards a longer length of stay for the early-week group compared with the late-week group, although this did not reach significance. The explanation for this trend is unclear but it may be due to low patient numbers involved or to the tendency to a longer length of stay due to patientrelated factors of emergency admissions.

\section{Discussion}

Overall, our results show a trend towards a longer length of stay for patients having surgery later in the week. This increase was significant for elective patients and for some types of surgery. There are many possible reasons for this difference, including the lack of physiotherapy and occupational therapy services soon after surgery and care by another team over the weekend less attuned to progressing patients towards discharge. Although we are not looking specifically at discharge over the weekend in this study, it is also possible that lack of community support over the weekend inhibits discharge of those who might otherwise have been ready for discharge at this point. Increased family support is likely to be available at weekend and would tend to mitigate this factor.

If the postoperative length of stay for the late-week group could be reduced to equal that of the early-week group, this would equate to a reduction in length of stay of 160 days over the course of the year. Focusing on femoral patients alone, this would account for a saving of 125 days. At an estimated cost of $£ 400$ per day for an inpatient, ${ }^{13}$ this 
Table 2 Comparison of median length of hospital stay for early and late periods separated by surgery type in days.

\begin{tabular}{llll} 
Surgery type & Early-week group (days) & Late-week group (days) & $\boldsymbol{P}$-value \\
Endovascular & $1(n=43)$ & $2(n=34)$ & 0.21 \\
Carotid endarterectomy & $1(n=49)$ & $1(n=32)$ & 0.34 \\
Femoral procedures & $2(n=52)$ & $4(n=50)$ & $<0.005$ \\
Endovascular aneurysm repair & $2(n=41)$ & $2(n=12)$ & 0.42 \\
Major amputation & $9(n=13)$ & $12.5(n=8)$ & 0.60 \\
Open abdominal aortic aneurysms & $5(n=21)$ & $7(n=11)$ & 0.06 \\
\hline
\end{tabular}

Table 3 Comparison of median length of hospital stay for early and late periods, separated by endovascular procedure type.

\begin{tabular}{llll} 
Endoscopic level procedure & Early-week group (days) & Late-week group (days) & $\boldsymbol{P}$-value \\
Iliac & $1(n=13)$ & $2(n=14)$ & 0.085 \\
Femoral & $1(n=7)$ & $2.5(n=4)$ & 0.41 \\
\hline Popliteal & $1.5(n=9)$ & $1(n=8)$ & 0.345 \\
Below knee & $1.5(n=7)$ & $3(n=3)$ & 0.12 \\
Vein bypass & $1(n=3)$ & $2(n=4)$ & 0.27 \\
Other & $1.5(n=4)$ & $1(n=1)$ & 0.8 \\
Multilevel & $2(n=10)$ & $1(n=7)$ & 0.475 \\
All procedures & $1(n=43)$ & $2(n=34)$ & 0.21 \\
\hline
\end{tabular}

would represent a significant saving, which would cover at least the cost of six hours of occupational therapy or physiotherapy time over the weekend.

Our study suggests that the strongest effect of operative timing is in those patients having a femoral artery procedure. Patients having carotid endarterectomy and endovascular procedures tend to stay in hospital for a short time and need little or no physiotherapy and occupational therapy input to get them mobile and home. Amputation patients can wait a significant amount of time for wheelchairs, transfer equipment and a definitive discharge destination. The operative procedures which tend to keep patients in for an intermediate length of time (femoral and possibly open aneurysm repair) are therefore more likely to be affected by an interruption to rehabilitation services over the weekend. The difference with open abdominal aneurysm repair is more difficult to explain, as mobility postoperatively should not be reliant on physiotherapy assistance. It is possible that this represents staff feeling cautious with progressing patients following major surgery due to reduced staffing available over the weekend. The reduction in discharges over the weekend could also cause delays in patients transferring from intensive care to the vascular ward if there are insufficient beds available, delaying discharge. There was no such relationship within emergency or urgent cases, which likely reflects the greater pathology in these patients, with longer recovery periods and increasing complex discharge arrangements.
The strength of this study is that it includes many patients with a wide pathology and procedure history. It is possible that the nature of case selection or operative techniques has some influence on the results but analysis of age and comorbidities and the fact that all teams use the Friday list argues against this. However, as this was a retrospective analysis it is difficult to quantify the impact of early- or late-week stays on length of hospital stay against many other confounding factors. For the comparison by operation types, the study also lacks the power to discern differences in some areas as the case numbers were too low for comparison. As our study recorded length of stay to the nearest 24 hours, it may overestimate the potential savings available and does not account for additional costs involved with community care. ${ }^{14}$ To further the work, we would like to see if a trial period of additional weekend support would reduce the variation in patient length of stay, particularly in patients undergoing infra-inguinal bypass procedures. It could also assess the impact that the additional weekend support has on patients with existing and subsequent tissue loss as this is associated with a particularly long stay in hospital.

\section{Conclusion}

This study has shown that the day of surgery may be impacting on vascular patients' length of hospital stay following surgery. Knowledge of this fact can allow for 
Table 4 Comparison of median length of hospital stay for early and late periods, by femoral procedure type for patients requiring no further surgery.

\begin{tabular}{llll} 
Surgery & Early-week group (days) & Late-week group (days) & $P$-value \\
Femoral endarterectomy & $2(n=27)$ & $4(n=22)$ & 0.007 \\
Femorofemoral bypass & $2(n=4)$ & $3(n=5)$ & 0.41 \\
Femoropopliteal bypass & $2(n=9)$ & $10(n=13)$ & $<0.005$ \\
Femorodistal bypass & $17(n=4)$ & $10(n=6)$ & 0.354 \\
All femoral procedures & $2(n=44)$ & $4(n=46)$ & $<0.005$ \\
\hline
\end{tabular}

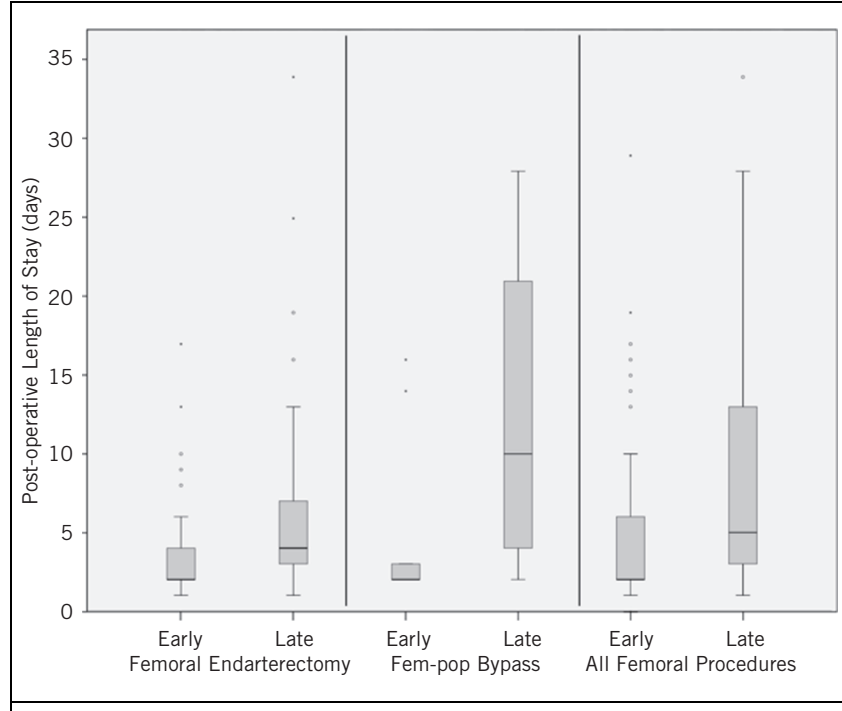

Figure 3 Comparison of early- and late-week groups for femoral procedures in patients undergoing a single operation.

improvement of the discharge pathway and staff availability at weekends to reduce this effect, freeing up bed spaces and helping ward efficiency. Equally, it could allow for lists to be planned over the week to minimise patients' length of stay. Further work is needed to assess the impact of a trial period of additional weekend support service staffing to see if this translates to a reduction in length of stay for those operated late in the week. A cost analysis could then be performed to see whether this adjustment would cover the costs involved in hiring extra staff at weekends.

\section{References}

1. Stowers MD, Lemanu DP, Hill AG. Health economics in enhanced recovery after surgery programs. Can J Anaesth 2015; 62: 219-230.

2. Feo CV, Portinari M, Tsolaki $\mathrm{E}$ et al. The effect of an enhanced recovery program in the elective retroperitoneal abdominal aortic aneurysm repair. J Vasc Surg 2016; 63: 888-894.

3. Greco M, Capretti G, Beretta L et al. Enhanced recovery program in colorectal surgery: a meta-analysis of randomized controlled trials. World J Surg 2014; 38: 1,531-1,541.

4. Reed T Jr, Veith FJ, Gargiulo NJ et al. System to decrease length of stay for vascular surgery. J Vasc Surg 2004; 39: 395-399.

5. Edirimanne $\mathrm{S}$, Roake JA, Lewis DR. Delays in discharge of vascular surgical patients: a prospective audit. A N Z J Surg 2010; 80: 443-446.

6. Han SM, Wu B, Eichler CM et al. Risk factors for 30-day hospital readmission in patients undergoing treatment for peripheral artery disease. Vasc Endovascular Surg 2015; 49: 69-74.

7. Sarkiss CA, Papin JA, Yao A et al. Day of surgery impacts outcome: rehabilitation utilization on hospital length of stay in patients undergoing elective meningioma resection. World Neurosurg 2016; 93: 127-132.

8. Newman JM, Szubski CR, Barsoum WK et al. Day of surgery affects length of stay and charges in primary total hip and knee arthroplasty. J Arthroplasty 2017; 32: 11-15.

9. Hijji FY, Narain AS, Haws BE et al. Does day of surgery affect hospital length of stay and charges following minimally invasive transforaminal lumber interbody fusion? Clin Spine Surg 2018; 31: E291-E295.

10. English C, Shields N, Brusco NK et al. Additional weekend therapy may reduce length of rehabilitation stay after stroke: a meta-analysis of individual patient data. J Physiotherapy 2016; 63: 124-129.

11. Parker AM, Lord RK, Needham DM. Increasing the dose of acute rehabilitation: is there a benefit? BMC Med 2013; 11: 199.

12. Medford AR, Agrawal S, Free CM, Bennett JA. Retrospective analysis of healthcare resource group coding allocation for local anaesthetic video-assisted 'medical' thoracoscopy in a UK tertiary respiratory centre. QJM 2009; 102: 329-333.

13. Department of Health. Reference costs 2015-16. London: DoH; 2016.

14. Jönsson B, Lindgren B. Five common fallacies in estimating the economic gains of early discharge. Soc Sci Med Med Econ 1980; 14: 27-33. 